\title{
Causal Role of Oxidants and Antioxidants in Atherosclerosis
}

\author{
Rachel Jacob ${ }^{1}$ \\ ${ }^{1}$ Department of Biochemistry, NIMS, Punjagutta, Hyderabad, \\ Telangana, India \\ Indian J Cardiovasc Dis Women-WINCARS 2018;00:4-5
}

Since 1989, when Steinberg et al first proposed that oxidation of low-density lipoprotein (ox-LDL) is the important initial step toward progression of atherosclerosis, ample evidence to support this hypothesis has been garnered. ${ }^{1}$

Oxidized LDL stimulates the endothelial cell to produce inflammatory markers, has cytotoxic effects on the endothelial cells, inhibits nitric oxide-induced vasodilatation, and promotes foam cell formation, all of which attenuate the process of atherosclerosis. Thus, oxidation of the lipids of LDL and apoprotein B-100 converts LDL into a proatherogenic molecule, while oxidation of high-density lipoprotein (HDL) impairs its inherent anti-atherogenic properties., ${ }^{2,3}$ Uptake of the ox-LDL by macrophages converts them into cholesterolladen foam cells, and this initiates the process of atherosclerosis, which progresses into vascular disease. ${ }^{4}$

Cells constantly generate reactive oxygen species and other free radicals, which function as signaling molecules and form an integral part of cellular homeostatic processes. These are balanced by the intra- and extracellular antioxidants. When the level of free radicals exceeds the antioxidant capacity, there is an oxidative stress, and these free radicals proceed to damage the lipids, proteins, and deoxyribonucleic acid (DNA) with deleterious effects on vascular structure and function. ${ }^{5}$

The cardiovascular antioxidant system is highlighted by the array of enzymatic and non-enzymatic factors, which help to reduce the cellular-free radicals by converting to less reactive form. Deficiency of these antioxidants will bring about oxidant stress, and there are many studies to support that systemic oxidant stress is associated with atherosclerosis. Inhibition of oxidation therefore, by use of antioxidants, should help to prevent atherosclerosis and related diseases. Several studies undertaken in animals and humans found the use of antioxidants in general to be effective in animal models. But large scale interventional studies in humans, using vitamin $\mathrm{E}$ as antioxidant, did not yield positive results, on the contrary, casting doubts on the oxidation hypothesis. ${ }^{6}$ Extensive studies on several oxidants responsible for oxidation of LDL such as metals (iron and copper), hemoglobin, and myoglobin, and enzymes such as myeloperoxidase, lipoxygenase, nicotinamide adenine dinucleotide phosphate (NADPH) oxidase; and NO synthase have confirmed that LDL may be oxidized in vivo by different mechanisms: either by free radical generation or by nonradical means. The lipid content in LDL is oxidized by several types of oxidants and differing mechanisms. A study of 114 healthy individuals without known atherosclerosis showed an inverse correlation between glutathione levels and carotid intima medial thickness. ${ }^{7}$

To supplement patients with antioxidants, initial measurements of antioxidant levels or their products and biomarkers of oxidative stress are done in either plasma or urine which is convenient and noninvasive. However, these levels fail to specify the actual location of oxidation, i.e., local site of atherogenesis or anywhere else in the body. However, the relation between the quantity of biomarkers and the clinical outcome in patients is unclear. In this issue, thte levels of oxidant/antioxidants in patients of acute coronary syndrome with left ventricular (LV) dysfunction who have undergone percutaneous intervention therapy and the correlation of oxidant and antioxidant levels with severity of LV dysfunction have been studied in an observational prospective study and are presented with the title "Oxidative and Anti-Oxidative Stress Markers in Relation to Left Ventricular dysfunction." ${ }^{\prime}$ Elevated malondialdehyde (MDA) levels were observed with severity of LV dysfunction, but for further correlation, larger population with continuous follow-up over a period of several years needs to be done.

Finally, it should be remembered that atherosclerosis is a chronic disease, and oxidation of LDL would necessarily occur many decades before the actual symptoms appear; hence, the levels of markers leading to oxidant stress should be constantly monitored over a long period to observe meaningful results.

\section{Address for correspondence}

Rachel Jacob, PhD, Retired Professor,

Department of Biochemistry, NIMS,

Punjagutta, Hyderabad, Telangana,

India (e-mail: rachel.jacob@gmail.com).
DOI https://doi.org/

10.1055/s-0038-1671702.
Copyright @2018 Women in Cardiology and Related Sciences
License terms

()(1) $\Theta$ 


\section{References}

1 Steinberg D, Parthasarathy S, Carew TE, Khoo JC, Witztum JL. Beyond cholesterol. Modifications of low-density lipoprotein that increase its atherogenicity. N Engl J Med 1989;320(14):915-924

2 Steinberg D. The LDL modification hypothesis of atherogenesis: an update. J Lipid Res 2009;50(Suppl):S376-S381

3 Shao B, Oda MN, Oram JF, Heinecke JW. Myeloperoxidase: an oxidative pathway for generating dysfunctional high-density lipoprotein. Chem Res Toxicol 2010;23(3):447-454

4 Miller YI, Choi SH, Fang L, Tsimikas S. Lipoprotein modification and macrophage uptake: role of pathologic cholesterol transport in atherogenesis. Subcell Biochem 2010;51:229-251
5 Leopold JA. Antioxidants and coronary artery disease: from pathophysiology to preventive therapy. Coron Artery Dis 2015;26(2):176-183

6 Niki E. Do free radicals play causal role in atherosclerosis? Low density lipoprotein oxidation and vitamin E revisited. J Clin Biochem Nutr 2011;48(1):3-7

7 Ashfaq S, Abramson JL, Jones DP, et al. The relationship between plasma levels of oxidized and reduced thiols and early atherosclerosis in healthy adults. J Am Coll Cardiol 2006;47(5):1005-1011

8 Garre I, Nallagasu R, Dangati MR, Bobbala I, Muddada R. Oxidative and anti-oxidative stress markers in relation to left ventricular dysfunction. Ind J Car Dis Wom 2018;3(1):6-11 